\title{
SOME EXPERIMENTAL AND CLINICAL OBSERVATIONS ON THE ANTICONVULSIVE ACTION OF PARALDEHYDE*
}

\author{
BY \\ F. J. DE ELfO, P. G. DE JALÓN and S. OBRADOR

\begin{abstract}
From the Instituto de Investigaciones Médicas, $†$ the Instituto de Farmacologia Experimental, $\neq$ and the Instituto de Neurocirugia,§ Madrid
\end{abstract}

In the practice of neurosurgical operations the convulsions occasionally elicited during cortical manipulations or produced by electrical stimulations may sometimes be difficult to control, and may give rise in some cases to dangerous convulsive states that may even end fatally. Barbiturates and other common drugs may fail to stop such convulsions quickly, as we have observed in some of our cases.

We have recently tried paraldehyde by intravenous injection in the treatment of such convulsive states, and the good results obtained have been the basis for an experimental and clinical study of this drug. In the literature we have found that Cervello (1884) was the first to use paraldehyde by mouth and rectum for narcosis. Nitzescu and Iacobovici (1934) recommended paraldehyde by intravenous injection $(0.15$ to $0.2 \mathrm{c.cm}$. per $\mathrm{kg}$.) as a basal anæsthetic before the administration of ether. Wechsler (1940) has also successfully employed the slow intravenous injection of $1 \mathrm{c.cm}$. of paraldehyde for the treatment of status epilepticus.

\section{Toxicity}

Paraldehyde has relatively little toxicity by the intravenous route. Experimentally $0.5 \mathrm{c.cm}$. per kg. in cats produces only a small and transitory reduction of the blood pressure and respiration. With smaller doses $(0 \cdot 1$ to $0.2 \mathrm{c.cm}$. per kg.) injected into rabbits, cats, and dogs there are no changes in respiration or circulation.

In human beings the intravenous injection of 1 to $3 \mathrm{c.cm}$. does not alter the pulse, blood pressure, or consciousness. There may be a slight fall in blood pressure lasting a few minutes. Frequently there is intense coughing for two to five minutes after the injection, with individual variations and caused by the irritation of the bronchial tree due to the partial elimination of the paraldehyde through the lungs. Usually there is also a stimulation of the respiration with hyperpnœa.

Higher doses of $6 \mathrm{c.cm}$. of intravenous paraldehyde in adults (about $0.1 \mathrm{c.cm}$. per $\mathrm{kg}$.) produce a narcotic effect with some diminution of conscious-

\footnotetext{
- Read before the Society of British Neurological Surgeons, Dublin Meeting, July, 1948.

†rof. C. Jimenez Diaz.

\& Dr. S. Obrador.
}

ness and a tendency to sleep, although the subject will still react to painful stimuli. There is also a slight fall in blood pressure. With bigger doses (9 to $12 \mathrm{c.cm}$. in adults) the narcotic action is more marked and usually the patients fall asleep at the end of the injection. The cough does not appear, the blood pressure falls slightly, and there is an increase in the rhythm and amplitude of the respirations.

\section{Action on the Motor Responses of the Cerebral Cortex}

In cats under dial anæsthesia the motor area was stimulated and the contractions of the contralateral tibialis anterior muscle were isometrically recorded. Condenser discharges of $10 \mathrm{~m} . \mathrm{sec}$. duration and with a frequency of 50 per second were applied through a bipolar electrode during periods of 5 seconds.

The same type of stimulation was employed in five patients submitted to neurosurgical operations, where the stimulation was necessary because of epileptic disorders or for delimitation of the motor area in cortical removals for athethosis.

Paraldehyde in small doses $(0.05$ to $0.1 \mathrm{c.cm}$. in cats and 1 to $3 \mathrm{c.cm}$. in men) produced a clear diminution of the motor responses of the cortex, with great elevation of the threshold of stimulation and even a complete abolition of the responses. Experimentally we have determined that the depressive action on the motor cortex lasted for 20 to 30 minutes, and afterwards the excitability recovered slowly although the diminution persisted for some time.

In some clinical cases the cortical stimulation produced a convulsive attack and the injection of paraldehyde stopped the convulsions immediately. Further stimulations were completely negative, and only with intense stimuli some slight clonic discharges were obtained in one epileptic patient.

\section{Action on the Electrical Activity of the Cerebral Cortex}

Cats under nembutal were used for recording the electrical activity of the cortex with electrodes inserted in the skull and in contact with the dura. A Grass electroencephalograph of four channels was employed for recording. 
The spontaneous activity of the cortex typical of the cat under nembutal with frequencies of 4 to 12 per second and 50 to 200 microvolts was not apparently modified by small doses of paraldehyde $(0.05 \mathrm{c.cm}$. per kg.). Greater doses $(0.1 \mathrm{c.cm}$. per kg.) produce, however, an obvious diminution of the spontaneous cortical activity that appears at 20 or 30 seconds from the injection time (Fig. 1). This effect lasts about 15 to 20 minutes, followed by slow recovery. Using higher amounts of paraldehyde $(0.15$ to $0.2 \mathrm{c.cm}$. per $\mathrm{kg}$.) the complete elimination of activity is very striking, as we shall see later (Fig. 3).

Paraldehyde also stops the convulsive activity of the cortex elicited by intravenous injection of leptazol (300 to $600 \mathrm{mg}$.). These convulsive discharges of very large voltage and high frequency appear a few seconds after leptazol injection, and paraldehyde abolishes such activity immediately (Fig. 2). The convulsive discharges may continue for several minutes in the control experiments. Sometimes paraldehyde in bigger doses $(0.2 \mathrm{c.cm}$. per kg.) not only stops the convulsive activity, bur may also abolish completely all signs of electricat activity in the brain (Fig. 3). This complete depression of the cortex lasted two minutes an afterwards some isolated discharges of spontaneous waves reappear, but it takes about 15 minutes before a marked recovery is noticed.

In several epileptic patients in the course of routine electroencephalographic studies, paralde? hyde was injected intravenously in small doses of: 2 to $3 \mathrm{c.cm}$. In some cases there was no noticeable change in the activity of the electroencephalogran? probably because of the small amount injected? But even these doses produced in two cases $\frac{D}{0}$ diminution of the abnormal activity. Furthes studies are still necessary, injecting larger amount of paraldehyde.

\section{Anticonvulsive Action}

The anticonvulsive action of paraldehyde has been studied experimentally in rabbits and cats.

A

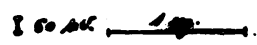

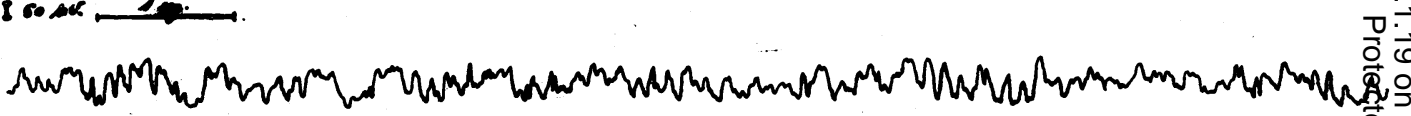

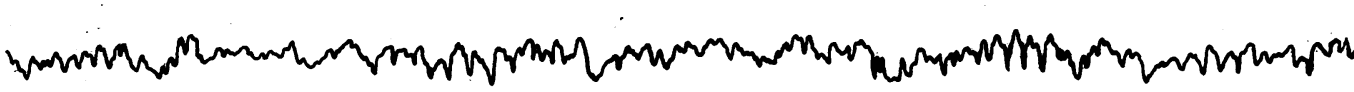

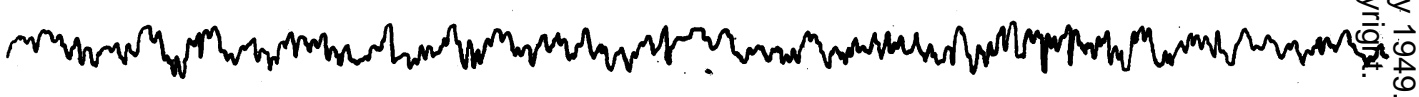

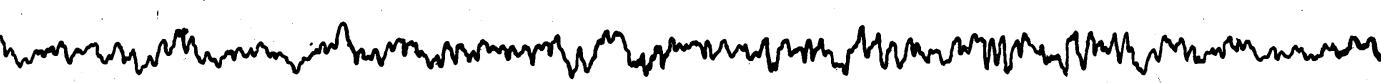
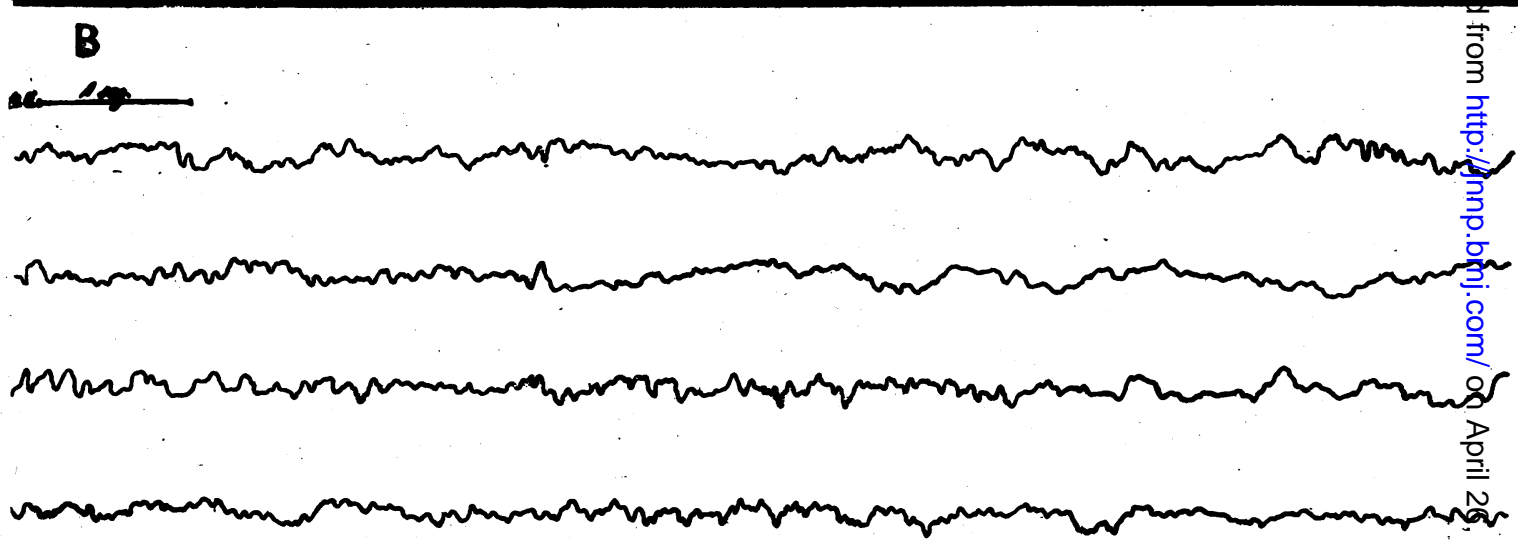

FIG. 1.-Electrical activity of a cat's cerebral cortex under Nembutal, before (A) and after (B) a small dose of paraldehyde $(0.1 \mathrm{c} . \mathrm{cm}$. per $\mathrm{kg}$.) 
This drug, in doses of 0.1 to $0.2 \mathrm{c.cm}$, is very effective in preventing and immediately stopping the very severe and lasting convulsions produced by large doses of leptazol and strychnine. The convulsions elicited by local applications of strychnine on the motor cortex of the cat under nembutal anxsthesia are also stopped to a striking degree by paraldehyde (Fig. 4). An animal injected with paraldehyde is completely protected even against very large doses of several $\mathrm{mg}$. of strychnine. The anticonvulsive action appears also in the decerebrate and spinal preparation of the cat.

Clinically we have also confirmed the intense anticonvulsive action of paraldehyde in several cases of epileptic attacks during or after neurosurgical operations. Even in cases under combined rectal avertin and intravenous pentothal anæsthesia we have seen convulsive fits during removal of scars or other cortical lesions that have quickly stopped with doses of 2 to $4 \mathrm{c.cm}$. of intravenous paraldehyde. Serious and prolonged status epilepticus has also been controlled by an injection of paraldehyde that sometimes had to be repeated. once or even twice in the course of several hours. We have never seen any complications or secondary effects after the use of paraldehyde.

\section{Other Pharmacological Actions}

The action of paraldehyde has also been studied on some pharmacological preparations. On the nerve and muscle preparation of the rat's diaphragm (Bülbring, 1946) this substance reduces the muscular responses to the electrical stimulation of the phrenic nerve (Fig. 5). The same depression has been observed on the nerve and muscle preparation of the sciatic nerve of the cat (Fig. 6). In the perfusion of the superior cervical sympathetic ganglion of the cat with the technique of Kibjakow (1933), modified by Feldberg and Gaddum (1934), the paraldehyde produces also a marked depression on the ganglionic synapse (Fig. 7).

\section{Discussion}

Our experimental and clinical observations on the effects of paraldehyde demonstrate very clearly the great depressive action of this drug on different

\section{A}

I jooser.

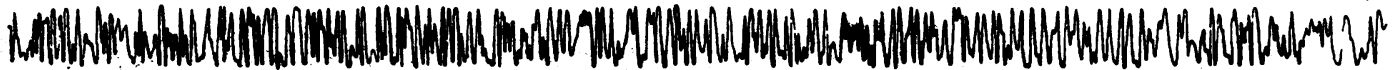
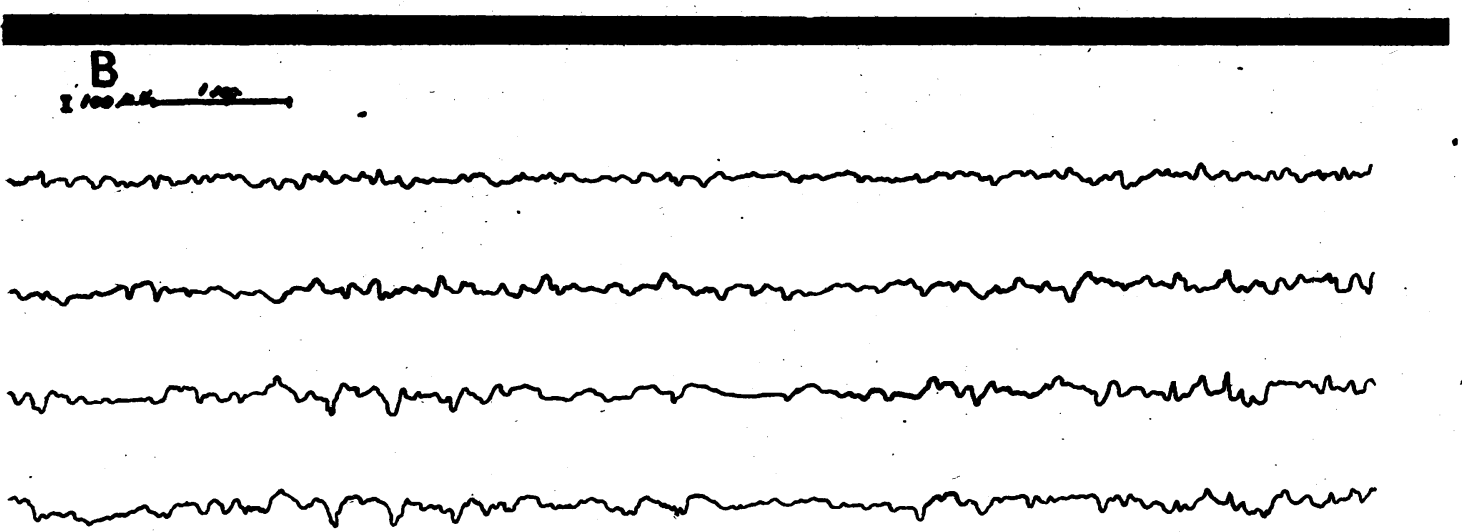

Fio. 2.-Convulsive activity of the cat's cortex after leptazol (A) abolished by paraldehyde (B). 
levels of the nervous system-peripheral, spinal, and cerebral. The action seems, however, more marked on the cerebral cortex, where paraldehyde can completely eliminate for several minutes all signs of electrical activity in the cat.

The mechanism of action of this substance is unknown to us, and in some preliminary experiments using the preparations of guinea-pig intestine and the rectus muscle of the frog we have seen that paraldehyde does not antagonize the action of acetylcholine.

From a theoretical point of view, the great anticonvulsive action of paraldehyde has some interest because of the similarity of this substance to other organic aldehydes that are normally formed in the complicated steps of the hydrocarbonate metabolism. Perhaps we may presume that paraldehyde acts something like other substances (glucose, carbon dioxide, pyruvic acid, etc.) that are considered as "physiological" anticonvulsants and regulators of the neuronal discharges. We hope to extend this line of investigation, comparing the anticonvulsive action of paraldehyde with other organic compounds and especially those related to the carbohydrate metabolism, although it is well known that the majority of the compounds that take a part in su\& metabolism are very unstable.

From a practical standpoint we believe that intravenous paraldehyde is actually the best age that we have to stop an epileptic discharge immedi ately. It can also be used as a prophylactic dru against operative convulsions in cortical operatios on epileptic patients. The small toxicity of the substance is a very important factor if a repetition of the injection is necessary. We recommend as first dose about $0.05 \mathrm{c.cm}$. to $0.1 \mathrm{c} . \mathrm{cm}$. per kg., ard if the convulsions reappear the injection may repeated one or two hours later. Although, as we have seen in our experimental studies, the marked action lasts only fifteen to thirty minutes, it is on necessary to inject the paraldehyde once to breas the epileptic state and usually the convulsions $\overline{d \theta}$

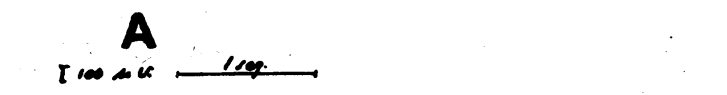

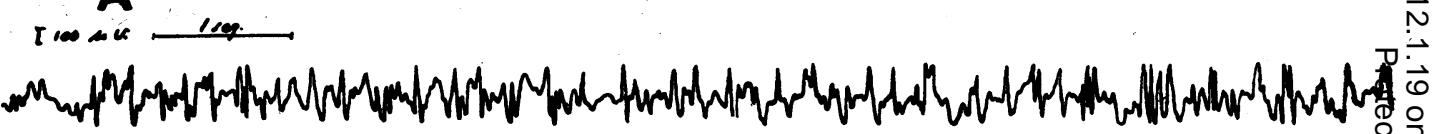

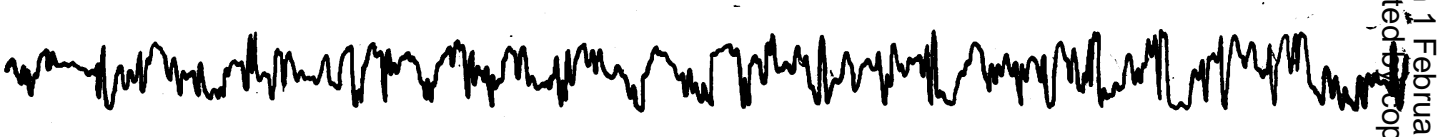

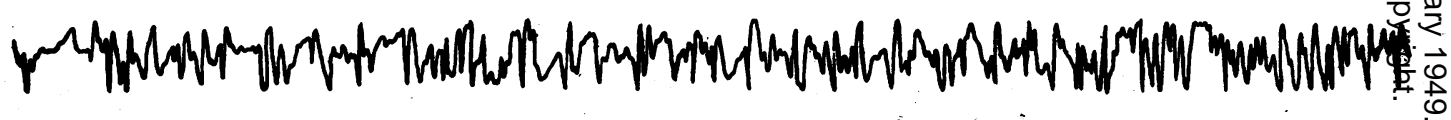

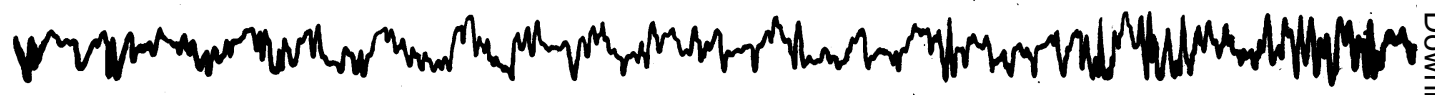

B Ines on

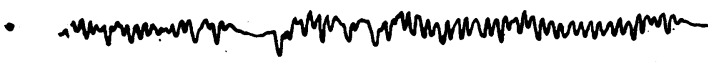

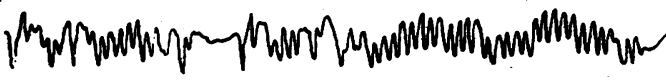

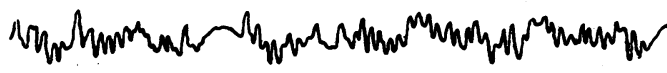

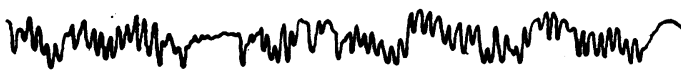

FIG. 3.-Convulsive activity (A) arrested by paraldehyde $(0 \cdot 2 \mathrm{c.cm}$. per $\mathrm{kg}$.$) , and all electrical activity als \tilde{\mathscr{C}}$ abolished (B). 


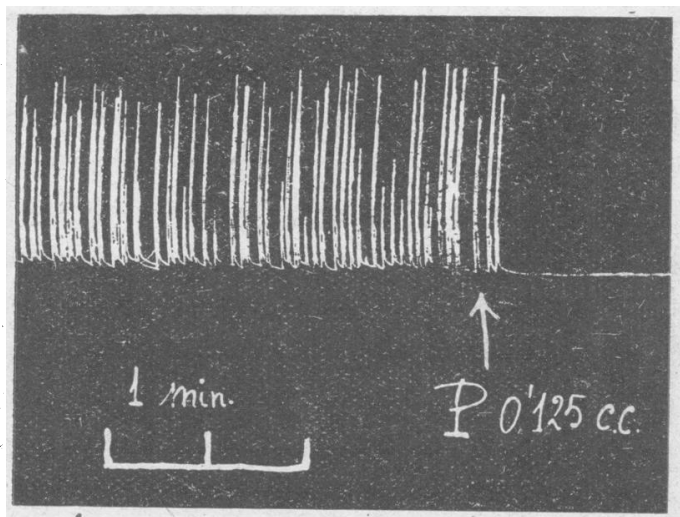

4

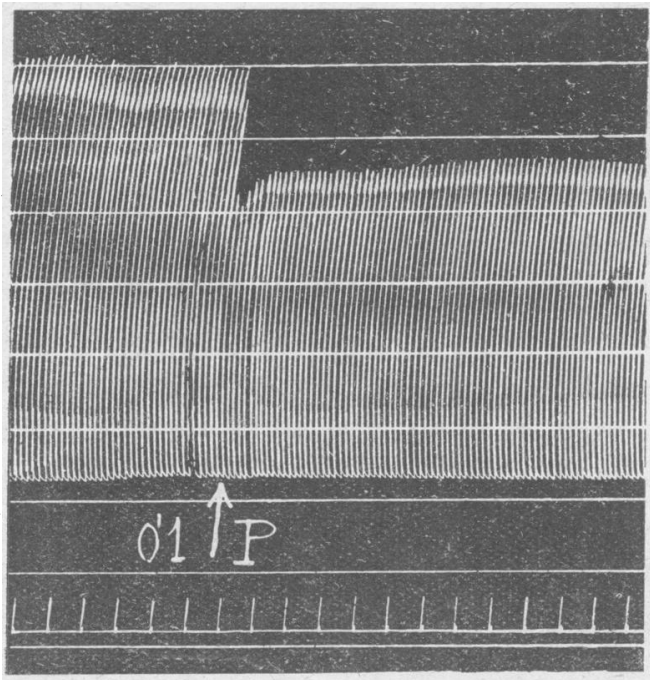

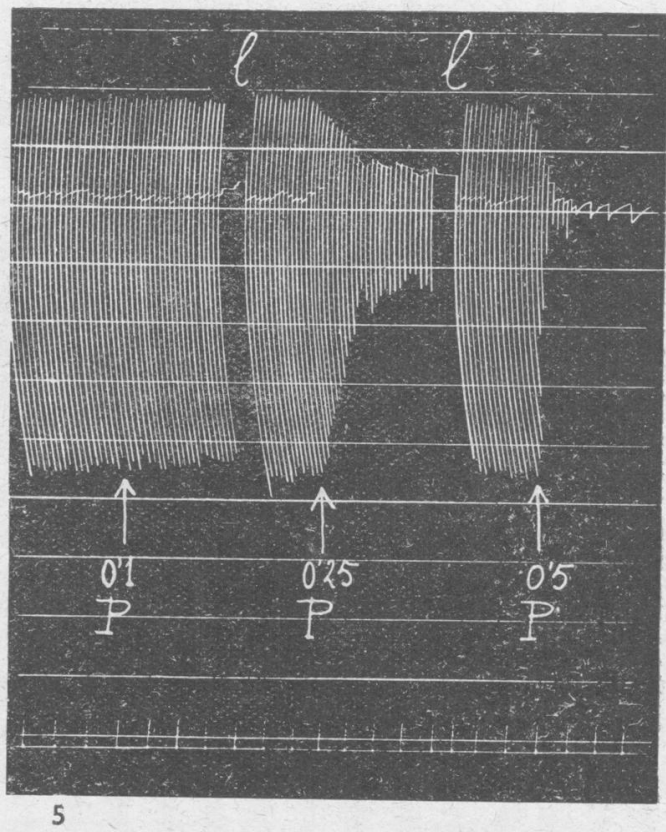

FIG. 4.-Electrocorticogram to illustrate the abolition of strychnine convulsions after the intravenous injection of paraldehyde at $\mathbf{P}$.

FIG. 5.-The effect of paraldehyde in reducing the muscular response of the isolated diaphragm of the rat to electrical stimulation of the phrenic nerve.

Fig. 6.-Contractions of the gastrocnemius muscle of the cat (under chloralose) elicited by stimulation of the sciatic nerve. Depression of contractions following the administration of paraldehyde at $\mathbf{P}$.

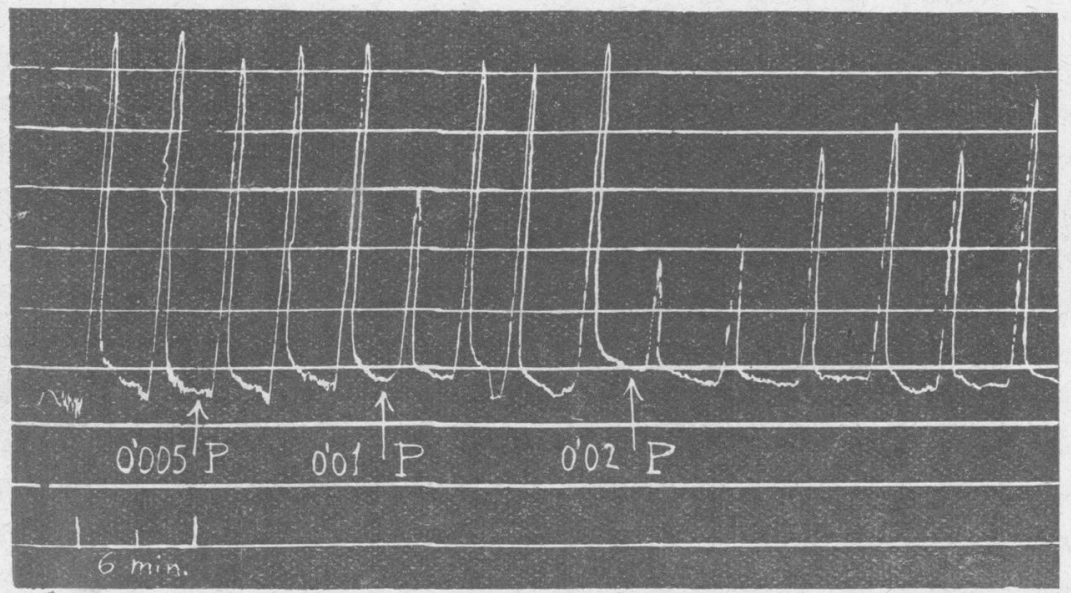

FIG. 7.-Superior cervical ganglion of the cat isolated and perfused with Locke solution. Stimulation of preganglionic fibres causing contraction of the nictitating membrane of the cat's eye. Depression of transmission of impulses by injection of paraldehyde into the fluid perfusing the ganglion at $\mathbf{P}$. 
not recur. An annoying side effect is the cough that follows the injection and that may last several minutes with great intensity in some individuals.

\section{Summary}

Paraldehyde is a substance with little toxicity that can be injected intravenously in doses of up to $0.2 \mathrm{c.cm}$. per $\mathrm{kg}$. without any ill-effects. Large doses have a narcotic action.

The motor responses of the cerebral cortex of the cat and human beings are greatly reduced or abolished by small doses of paraldehyde. The convulsive attacks produced in the course of neurosurgical operations by cortical stimulation are immediately stopped by paraldehyde.

Paraldehyde reduces or abolish for some minutes the spontaneous cortical activity of the cat according to the dose employed. Convulsive discharges of the cortex elicited by leptazol disappear immediately with the injection of paraldehyde.

The anticonvulsive action is also very striking in animals injected with different convulsant drugs. A dose of paraldehyde protects an animal against large doses of strychnine and leptazol. The anti- convulsive action appears also in the decerebrate and spinal preparations. Clinically, paraldehy has been used in many cases to stop operative or postoperative convulsions that in some cases may reach serious proportions.

The depressing action of paraldehyde has also been demonstrated on the peripheral neuromuscular. junctions and on the ganglion synapses, although the action is more marked on the cerebral cortex 2

The theoretical implications and mechanism of action of paraldehyde are briefly discussed. This substance is recommended in doses of 0.05 $0.1 \mathrm{c.cm}$. per $\mathrm{kg}$. intravenously as the best age that we actually have to break and stop a convulsife discharge. The dose may be repeated if necessar

\section{REFERENCES}

œ

Building, E. (1946). British J. Pharm. Chem., 1, 38. $\vec{\circ}$ Cervello, V. (1884). From Adriani, J., " The Chemisty of Anæsthesia." (1946). Oxford.

Feldberg, W., and Gaddum, J. H. (1934). J. Physi尔, 81, 305 .

Kibjakow, A. W. (1933). Pfüg. Arch., 232, 432.

Nitzescu, I. I., and lacobovici, I. (1934). Presse méd Wechsler, I. S. (1940). J. Amer. med. Ass., 114, 2198. 